\title{
Comparison of Hyperfractionated Radiotherapy with Concomitant Chemotherapy and Conventional Chemoradiotherapy in Locally Advanced Head and Neck Cancer Treated with Volumetric Modulated Arc Technique (VMAT)
}

\section{Chatakonda S Snehit ${ }^{1}$, Bhaskar Vishwanathan ${ }^{1}$, Geeta S Narayanan ${ }^{1}$, Shashidhar V Kapurmath ${ }^{2}$, Vandana S Kushwaha ${ }^{1 *}$, Sowmya S Narayanan $^{3}$ and Ramya Venugopal ${ }^{3}$}

${ }^{1}$ Department of Radiation Oncology, Vydehi Institute of Medical Sciences and Research Centre, Bangalore, Karnataka, India

${ }^{2}$ Department of Medical Oncology, Vydehi Institute of Medical Sciences and Research Centre, Bangalore, Karnataka, India

3Department of Radiation Physics, Vydehi Institute of Medical Sciences and Research

Centre, Bangalore, Karnataka, India

*Corresponding Author: Vandana S Kushwaha, Department of Radiation Oncology, Vydehi Institute of Medical Sciences and Research Centre, Bangalore, Karnataka, India.
Received: March 20, 2021

Published: April 09, 2021

(C) All rights are reserved by Vandana $\mathbf{S}$

Kushwaha., et al.

\section{Abstract}

Background: Concomitant Chemoradiotherapy (CRT) is standard approach for treating locally advanced head and neck squamous cell carcinoma (HNSCC). Altered fractionation such as hyperfractionated radiotherapy (HF) has also shown similar survival benefits as compared to conventional CRT. To determine whether addition of concomitant chemotherapy to hyperfractionated radiotherapy further improves outcome in HNSCC patients treated with modern volumetric arc modulated radiotherapy (VMAT).

Methods: From January 2018 to March 2019, thirty four patients with stage III-IV HNSCC (excluding oral cavity, nasopharynx and paranasal sinus) were randomly assigned to hyperfractionated HFCRT (VMAT) with concomitant chemotherapy arm with radiation dose 81.6Gy/68 fractions,1.2Gy/fraction, twice daily, 5days/week or Conventional CRT (VMAT) arm with radiation dose $70 \mathrm{GY} / 35$ fractions, 2Gy/fraction, once daily, 5days/week, seventeen patients in each arm. Concurrent cisplatin $40 \mathrm{mg} / \mathrm{m}^{2} /$ week was given in both arm from first week. Overall survival (OS), Disease free survival (DFS) and toxicities were assessed.

Results: The cisplatin full dose was received by $94.1 \%$ and $82.4 \%$ patients in HFCRT (VMAT) and CRT (VMAT) arm respectively. High grade (3-4) acute skin and mucosal were higher in HFCRT (VMAT) arm (47.1\%) in comparison to CRT (VMAT) arm (17.6\%) with no statistical significance $(\mathrm{p}=0.067)$. Late toxicity was similar in both arms. The OS was better in HFCRT (VMAT) arm with median overall survival-17 months as compared to CRT (VMAT) arm with median overall survival -12 months, however, difference did not reach significance (log-rank test, $\mathrm{p}=0.341$ ), with similar trend in DFS.

Conclusion: Hyperfractionated HFCRT (VMAT) with concomitant chemotherapy showed an encouraging trend for OS and DFS with acceptable toxicity profile. A larger study with longer follow up needed to elucidate possible superiority of HFCRT over CRT.

Keywords: Hyperfractionated Radiotherapy; Volumetric Arc Modulated Therapy (VMAT); Concomitant; Concurrent Chemoradiotherapy; Head and Neck Cancer; Squamous Cell Carcinoma

Citation: Vandana S Kushwaha. "Comparison of Hyperfractionated Radiotherapy with Concomitant Chemotherapy and Conventional Chemoradiotherapy in Locally Advanced Head and Neck Cancer Treated with Volumetric Modulated Arc Technique (VMAT)". Acta Scientific Cancer Biology 5.5 (2021): 02-11. 
Comparison of Hyperfractionated Radiotherapy with Concomitant Chemotherapy and Conventional Chemoradiotherapy in Locally Advanced Head and Neck Cancer Treated with Volumetric Modulated Arc Technique (VMAT)

\section{Introduction}

Head and neck squamous cell carcinoma (HNSCC) is among the common five leading cancers in India, accounting for $35.1 \%$ of all cancer in males and $8.9 \%$ in females according to report from $\mathrm{Na}$ tional Cancer Registry Programme (2012-2016) [1]. The majority of the patients were diagnosed at the locally advanced stages with poor 5-year survival rate $(40 \%)$ with all modalities of treatment [2]. Numerous studies have been done to improve locoregional control and survival in patients with HNSCC, by adding chemotherapy or targeted agents to radiation or altering fractionation size of radiation. Combined concomitant chemoradiation (CRT) has shown significantly improved overall survival (absolute benefit 6.5\% at 5 years) than radiation alone and become the standard of care in patients with locally advanced HNSCC [3]. Altered fractionation such as hyperfractionated radiotherapy (HFRT) have also shown similar benefits (absolute difference at 5 years of $8 \cdot 1 \%$ ) over conventional fractionation [4].

Addition of concurrent chemotherapy to hyperfractionated radiotherapy (HFCRT) whether feasible with acceptable toxicity or have any benefit over hyperfractionated radiation (HFRT) alone, have been investigated in numerous studies [5-7]. A recent metaanalysis comparing hyperfractionated radiotherapy (HFRT) alone to hyperfractionated radiotherapy with concurrent chemotherapy (HFCRT) have shown significant improvement in overall survival and locoregional recurrence without significant increase in highgrade toxicities [8]. However, variable chemotherapy scheduling and dosing with different radiation dose in the meta-analysis was used, so the most effective combination of chemotherapy and radiotherapy has yet to be defined.

Although chemoradiation (CRT) or hyperfractionated radiotherapy (HFRT) has shown improved efficacy but at the expense of higher rates of treatment related toxicity as compared to conventional fractionated radiotherapy alone. Both the treatment regimens are associated with increased risk of severe acute skin and mucosal reactions [9]. Even late reactions such as xerostomia and swallowing dysfunction are also major problems with these regimens using conventional radiation techniques [10]. With the advances in Linear accelerator and the advent of Intensity modulation radiation technique (IMRT), escalated dose can be delivered to target volume while reducing the dose to surrounding normal tissue and sparing Organs at risk (OAR), thereby causing less treatment related toxicity and higher therapeutic index [11]. As a fur- ther evolution, new technique Volumetric modulated arc therapy (VMAT) has been introduced which enables delivery of IMRT like dose distributions from a continuous rotation of the gantry. VMAT can achieve highly conformal dose distributions with improved target volume coverage and sparing of normal tissues and decreases treatment delivery time compared to conventional IMRT. To date, limited studies reported the clinical experience of treatment of HNSCC with VMAT and they showed improved treatment outcome with good toxicity profile $[12,13]$.

In designing this study authors hoped to maintain high rates of local control and survival while reducing toxicity by incorporating modern intensity modulation technique VMAT for delivering radiation. The purpose of the study is to determine whether addition of concomitant chemotherapy to hyperfractionated radiotherapy further improves outcome as compared to conventional chemoradiation. The current study is the only report in the medical literature to our knowledge that has compared hyperfractionated radiotherapy with concomitant chemotherapy (HFCRT) to conventional chemoradiotherapy (CRT) using VMAT in locally advanced HNSCC.

\section{Methods}

We conducted a prospective randomized study from January 2018 to March 2019 in histologically proven head and neck squamous cell carcinoma assigned to receive chemoradiation in our institute. The study was approved by the Vydehi Institutional Ethics Committee and all patients provided written informed consent for their participation in the study.

\section{Eligibility}

Patient inclusion criteria were unresectable HNSCC, stage III - IVB, age group of 18 - 65 years, Eastern Co-operative Oncology Group (ECOG) performance status grade 0-2, Haemoglobin level > $10 \mathrm{mg} / \mathrm{dl}$, Total Leukocyte count (TLC) $>4000 / \mathrm{mm}^{3}$, platelet count $>100000 / \mathrm{mm}^{3}$, adequate serum creatinine $0.6-1.5 \mathrm{mg} / \mathrm{dl}$ and normal electrocardiogram (ECG). Exclusion criteria were carcinoma of oral cavity, nasopharynx, nasal cavity and paranasal sinuses and prior history of cancer, or synchronous malignancy. Patients were clinically evaluated, and Staging was done as per AJCC cancer staging system $8^{\text {th }}$ edition 2017 [4]. Radiological assessment was done in each patient with contrast enhance computed tomography (CECT) of Head and Neck region, and chest X-ray posteroanterior (PA) view. 


\section{Treatment details}

Patients enrolled in the study were randomized into two treatment arms- ARM1 and ARM2 by computer generated random table number (Figure 1).

- $\quad$ ARM1: HFCRT (VMAT)-Patients were given hyperfractionated radiotherapy total dose of $81.6 \mathrm{~Gy} / 68 \mathrm{fr}$ over 7 weeks (1.2 Gy/fraction, twice daily (6 hours gap), 5 days/week) to gross primary and nodal disease with concurrent cisplatin $40 \mathrm{mg} / \mathrm{m}^{2}$ per week.

- $\quad$ ARM2: CRT (VMAT)- Patients were given conventional radiotherapy total dose of 70 Gy in 35 fractions over 7 weeks ( 2 Gy/fraction, 5 days in a week) to gross primary and nodal disease along with concurrent cisplatin $40 \mathrm{mg} / \mathrm{m}^{2}$ per week.
Immobilization was done via 5 fixation point thermoplastic mask to ensure minimal motion during simulation and treatment. Planning CT of section thickness $2.5 \mathrm{~mm}$. were acquired from vertex to T4 vertebrae. Diagnostic contrast enhanced CT was coregistered with planning CT for contouring. General definitions of gross tumor volume (GTV), clinical target volume (CTV), and planning target volume (PTV) were followed according to International Commission on Radiation Units and Measurements guidelines ICRU 83. Hyperfractionated VMAT RT with targets of PTV 1, PTV 2 and PTV 3, being prescribed with 81.6 Gy (1.2Gy/Fr), 69.6 Gy (1.2Gy/Fr) and 60 Gy (1.2Gy/Fr), twice daily (6 hours gap),respectively and Conventional VMAT RT with targets of PTV 1, PTV 2 and PTV 3, being prescribed with $70 \mathrm{~Gy}$ (at 2Gy/Fr), $60 \mathrm{~Gy}$ (at 2Gy/Fr) and $50 \mathrm{~Gy}$ (at $2 \mathrm{~Gy} / \mathrm{Fr}$ ), respectively. Treatment was delivered via sequential boost.

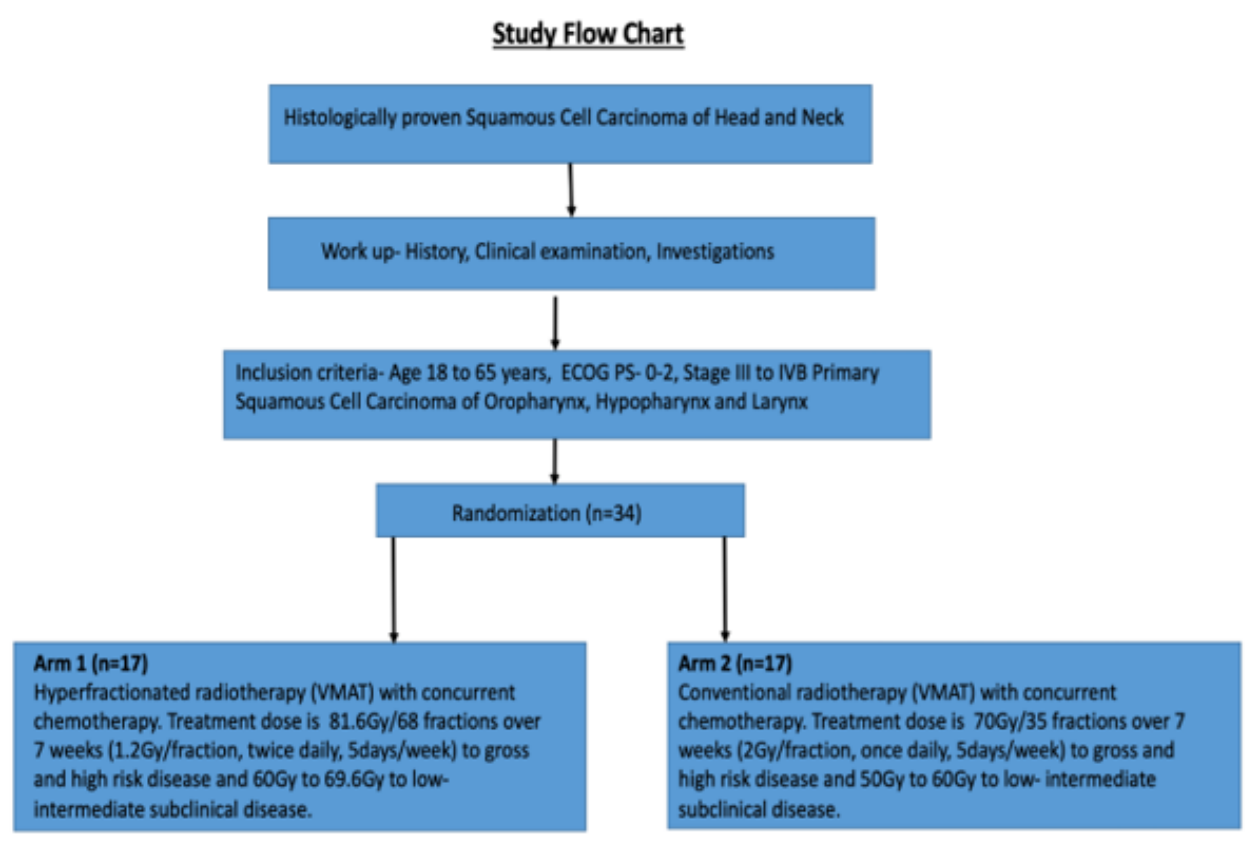

Figure 1: Flow chart of study design.

All VMAT plans were optimized on the Eclipse Treatment Planning System version 11 in its RapidArc form. VMAT double arc plans using complementary coplanar arcs of $360^{\circ}$ (one counterclockwise from $179^{\circ}$ to $181^{\circ}$, one clockwise from $181^{\circ}$ to $179^{\circ}$ ) were done. Progressive Resolution Optimization (PRO) algorithm was used for inverse planning and dose was calculated using AAA.
Plan objectives were: $95 \%$ of prescribed dose to cover $95 \%$ of PTV $\left(\mathrm{V}_{95 \%}>95 \%\right)$ and near to maximum dose to high risk PTV $\left(\mathrm{D}_{2 \%}\right)$ below $105 \%$ of prescribed dose. Plans were optimized according to requirements for target volume coverage and dose limits for Organ at risk (OAR). International Commission on Radiation Units and Measurements guidelines (ICRU 83 report) were applied. 
Study assessment

Response to treatment was assessed after 3 months of treatment completion in both the arms. Response evaluation was done by clinical assessment with imaging and biopsy or cytology was done when required. RECIST (Response Evaluation Criteria In Solid Tumours) version 1.1 was used for response assessment [15].

Patients were assessed in every week during treatment for acute toxicities. Weekly Hemogram and complete blood biochemistry for renal function were done in all patients. Late toxicity was recorded as any toxicities post 3 month of treatment. Acute Radiation Morbidity Criteria and RTOG/EORTC (European Organisation for Research and Treatment of Cancer) Late Radiation Morbidity Criteria were used for toxicity assessment [16].

Primary endpoints was to compare treatment response, disease free survival (DFS), and overall survival (OS) rates among the treatment arms. Secondary endpoints were to compare compliance to treatment and treatment related toxicities between two arms. Pattern of failure and recurrence were also studied.

\section{Statistical consideration}

The statistical analysis was done using SPSS 22.0 (Statistical Package for Social Sciences), and R environment ver.3.2.2 were used for the analysis of the data. The values were represented in Number (\%) and Mean \pm SD. The Chi-square/ Fisher Exact test has been used to find the significance of study parameters on categorical scale between two or more groups. Survival between the arms was done by Kaplan-Meier method using Log rank test. A 2-sided $P$ value less than $0.05(\mathrm{P}<0.05)$ was considered statistically significant.

\section{Results}

Patients population

A total of 34 patients enrolled in the study and randomized in the two arms, seventeen patients in each arm. The basic demographic data and clinical characteristics of two arms are presented in table 1 . The treatment arms were well balanced with respect to age, gender, smoking habits, primary site and tumor grade, Majority of the patients had oropharynx followed by larynx as primary disease site. Most of the patients in HFCRT (VMAT) arm has T4 tumor stage $(47.1 \%)$ and N2/N3 nodal stage (58.8\%) while in CRT (VMAT) arm, limited T4 (11.7\%) and N2/N3 (5.8\%) patients were present $(\mathrm{p}=0.020, \mathrm{p}=0.019$ respectively). Patients with advanced stage IV (76.5\%) were significantly higher in HFCRT (VMAT)arm as compared to CRT (VMAT) arm where 5 (29.4\%) patients were stage IV ( $p=0.022$ ). Sixteen (94.1\%) patients in HFCRT (VMAT) arm and 14 (82.4\%) patients in CRT (VMAT) arm completed 6-7 weekly cycles of cisplatin as per protocol ( $p=0.601$ ). The median overall treatment time (OTT) was 53 days in both arm with range (45-64) days in HFCRT (VMAT) arm and (49-60 days) in CRT (VMAT)arm. All the patients in both arms completed their treatment as per protocol with some treatment interruptions.

\section{Toxicity}

Acute toxicities as low and high grade are listed in table 2. Skin toxicities (Grade III and IV) were seen relatively more in HFCRT (VMAT) arm than CRT (VMAT) arm during treatment (47.1\% vs $17.6 \%)$ with no statistical significance ( $p=0.067)$. Similarly, mucositis (Grade III and IV) were more in HFCRT (VMAT) arm than CRT (VMAT) arm during treatment (47.1\% vs $17.6 \%$ ) with no statistical significance $(\mathrm{p}=0.067)$. Hematological toxicities (Grade III and IV ) was seen in $23.5 \%$ patients in both arms during treatment ( $p=$ 0.999). Dysphagia (Grade III and IV) was seen more in CRT (VMAT) arm than HFCRT (VMAT) (29.4\% vs $17.6 \%$ ) during treatment but statistical significance was not achieved $(p=0.419)$.

No grade III and IV late toxicities of skin, mucosal, dysphagia, xerostomia and laryngeal edema were noted during follow up in both arms. In HFCRT (VMAT) arm, 9 (52.9\%) patients, 5 (29.4\%) patients and 6 (35.3\%) patients while in CRT (VMAT) arm, 7 $(41.2 \%)$ patients, $4(23.5 \%)$ patients and $7(41.2 \%)$ patients developed Grade I skin, mucosal and dysphagia respectively with no statistical significance ( $\mathrm{p}>0.05)$. Xerostomia (Grade I/II) was seen more in HFCRT (VMAT) arm than CRT (VMAT) arm (41.2\% vs $35.3 \%)$ on follow up ( $\mathrm{p}=0.719)$. Two $(11.76 \%)$ patients presented with Grade I laryngeal edema in each arm.

\section{Response}

The response to therapy is summarized in table 3. At the end of 3 months follow-up, 12 (70.6\%) patients achieved complete response (CR), 2 (11.8\%) patients had partial response (PR), 2 (11.8\%) patients had stable disease (SD) and in 1 (5.9\%) patients disease progressed in HFCRT (VMAT) arm whereas in CRT (VMAT) arm, $9(52.9 \%)$ patients, $3(17.6 \%)$ patients, 4 (23.5\%)patients and $1(5.9 \%)$ had complete response (CR), partial response (PR), stable disease (SD) and progressive disease (PD) respectively. The proportion of patients with CR were more in HFCRT (VMAT) arm as compared to CRT (VMAT) arm, however, it didn't reach statistical significance $(\mathrm{p}=0.788)$. 


\begin{tabular}{|c|c|c|c|}
\hline Variables & $\begin{array}{c}\text { ARM } 1 \\
\text { HFCRT (VMAT) } \\
\mathbf{n}=\mathbf{1 7}\end{array}$ & $\begin{array}{c}\text { ARM } 2 \\
\text { CCRT (VMAT) } \\
\text { n = } 17\end{array}$ & $P$ value \\
\hline Age $($ Mean \pm SD) & $49.11 \pm 14.27$ & $49.11 \pm 14.27$ & $\mathrm{P}=0.245$ \\
\hline \multicolumn{4}{|l|}{ Gender } \\
\hline Female & 2 (11.8\%) & $2(11.8 \%)$ & \\
\hline Male & $15(88.2 \%)$ & 15 (88.2\%) & $P=1.000$ \\
\hline \multicolumn{4}{|l|}{ Tobacco Chewing } \\
\hline No & $4(23.5 \%)$ & $12(70.6 \%)$ & $P=0.006^{*}$ \\
\hline Yes & $13(76.5 \%)$ & $5(29.4 \%)$ & \\
\hline \multicolumn{4}{|l|}{ Smoking } \\
\hline No & $5(29.4 \%)$ & $4(23.5 \%)$ & $P=0.697$ \\
\hline Yes & $12(70.6 \%)$ & $13(76.5 \%)$ & \\
\hline \multicolumn{4}{|l|}{ Primary Site } \\
\hline Oropharynx & $10(58.8 \%)$ & $6(35.3 \%)$ & \\
\hline Hypopharynx & $3(17.6 \%)$ & $5(29.4 \%)$ & \\
\hline Larynx & $4(23.5 \%)$ & $6(35.3 \%)$ & $P=0.491$ \\
\hline \multicolumn{4}{|l|}{ HPE } \\
\hline WDSCC & $3(17.6 \%)$ & $7(41.2 \%)$ & \\
\hline MDSCC & $12(70.6 \%)$ & $7(41.2 \%)$ & \\
\hline PDSCC & $2(11.8 \%)$ & $3(17.6 \%)$ & $P=0.256$ \\
\hline \multicolumn{4}{|l|}{ Tumor Staging } \\
\hline 2 & $0(0 \%)$ & $3(17.6 \%)$ & \\
\hline 3 & $8(47.1 \%)$ & $12(70.6 \%)$ & \\
\hline 4 & $9(52.9 \%)$ & $2(11.8 \%)$ & $P=0.02^{*}$ \\
\hline \multicolumn{4}{|l|}{ Nodal Staging } \\
\hline 0 & $2(11.8 \%)$ & $6(35.2 \%)$ & \\
\hline 1 & $5(29.4 \%)$ & $9(52.9 \%)$ & $P=0.019^{*}$ \\
\hline 2 & $9(52.9 \%)$ & $2(11.8 \%)$ & \\
\hline 3 & $1(5.9 \%)$ & $0(0 \%)$ & \\
\hline \multicolumn{4}{|l|}{ AJCC Staging } \\
\hline III & $4(23.5 \%)$ & $12(70.5 \%)$ & \\
\hline IVA & $11(64.7 \%)$ & $5(29.4 \%)$ & $\mathrm{P}=0.022^{*}$ \\
\hline IVB & $2(11.8 \%)$ & $0(0 \%)$ & \\
\hline \multicolumn{4}{|l|}{ Chemotherapy } \\
\hline Yes & $16(94.1 \%)$ & $14(82.4 \%)$ & \\
\hline No & $1(5.9 \%)$ & $3(17.6 \%)$ & $P=0.601$ \\
\hline \multicolumn{4}{|l|}{ OTT } \\
\hline Mean \pm SD & $52.64 \pm 7.47$ & $53.70 \pm 3.21$ & \\
\hline Median & $53(45-64)$ & $53(49-60)$ & $P=0.595$ \\
\hline
\end{tabular}

Table 1: Patient demographic and clinical characteristics.

HFCRT: Hyperfractionated Chemo-radiotherapy; CCRT: Concomitant Chemo-radiotherapy; SD: Standard Deviation; HPE: Histopathological Examination; WDSCC: Well Differentiated Squamous Cell Carcinoma; MDSCC: Moderately Differentiated Squamous Cell Carcinoma; PDSCC: Poorly Differentiated Squamous Cell Carcinoma; OTT: Overall Treatment Time; *: Statistically Significant. 


\begin{tabular}{|c|c|c|c|}
\hline Variables & $\begin{array}{c}\text { ARM 1 } \\
\begin{array}{c}\text { HFCRT (VMAT) } \\
\text { n = } 17\end{array}\end{array}$ & $\begin{array}{c}\text { ARM } 2 \\
\text { CCRT (VMAT) } \\
\text { n = } 17\end{array}$ & $P$ value \\
\hline Skin & & & \\
\hline $\begin{array}{l}\text { Low grade (I-II) } \\
\text { High Grade (III-IV) }\end{array}$ & $\begin{array}{l}9(52.9 \%) \\
8(47.1 \%)\end{array}$ & $\begin{array}{l}14(82.4 \%) \\
3(17.6 \%)\end{array}$ & 0.067 \\
\hline Mucosal & & & \\
\hline $\begin{array}{l}\text { Low grade (I-II) } \\
\text { High Grade (III-IV) }\end{array}$ & $\begin{array}{l}9(52.9 \%) \\
8(47.1 \%)\end{array}$ & $\begin{array}{c}14(82.4 \%) \\
3(17.6 \%)\end{array}$ & 0.067 \\
\hline $\begin{array}{l}\text { Dysphagia } \\
\text { Low grade (I-II) } \\
\text { High Grade (III-IV) }\end{array}$ & $\begin{array}{c}14(82.4 \%) \\
3(17.6 \%)\end{array}$ & $\begin{array}{l}12(70.6 \%) \\
5(29.4 \%)\end{array}$ & 0.419 \\
\hline $\begin{array}{l}\text { Hematological } \\
\text { Low grade (I-II) } \\
\text { High Grade (III-IV) }\end{array}$ & $\begin{array}{l}14(82.4 \%) \\
4(47.1 \%)\end{array}$ & $\begin{array}{l}14(82.4 \%) \\
4(23.5 \%)\end{array}$ & 1.000 \\
\hline
\end{tabular}

Table 2: Acute toxicity.

\begin{tabular}{|c|c|c|c|}
\hline Variables & $\begin{array}{c}\text { ARM } 1 \\
\text { HFCRT (VMAT) } \\
\text { n = 17 } \\
\end{array}$ & $\begin{array}{c}\text { ARM } 2 \\
\text { CCRT (VMAT) } \\
\text { n = } 17\end{array}$ & $P$ value \\
\hline \multicolumn{4}{|l|}{ Response at 3 months } \\
\hline $\mathrm{CR}$ & $12(70.6 \%)$ & $9(52.9 \%)$ & \\
\hline PR & $2(11.8 \%)$ & $3(17.6 \%)$ & 0.788 \\
\hline SD & $2(11.8 \%)$ & $4(23.5 \%)$ & \\
\hline PD & $1(5.9 \%)$ & $1(5.9 \%)$ & \\
\hline \multicolumn{4}{|l|}{ Response at 6 months } \\
\hline $\mathrm{CR}$ & $13(76.5 \%)$ & $11(64.7 \%)$ & \\
\hline PR & $1(5.9 \%)$ & $0(0 \%)$ & 0.235 \\
\hline SD & $1(5.9 \%)$ & $0(0 \%)$ & \\
\hline PD & $2(11.8 \%)$ & $6(35.3 \%)$ & \\
\hline \multicolumn{4}{|l|}{ Recurrence } \\
\hline No & $14(82.4 \%)$ & $13(76.5 \%)$ & 0.995 \\
\hline Yes & $3(17.6 \%)$ & $4(23.5 \%)$ & \\
\hline Local recurrence & $2(11.8 \%)$ & $2(11.8 \%)$ & \\
\hline Distant recurrence & $1(5.9 \%)$ & $2(11.8 \%)$ & \\
\hline \multicolumn{4}{|l|}{ Patient status at last follow up } \\
\hline Alive & $10(58.8 \%)$ & $8(47.1 \%)$ & 0.492 \\
\hline Deceased & $7(41.2 \%)$ & $9(52.9 \%)$ & \\
\hline \multicolumn{4}{|l|}{ Follow up (months) } \\
\hline Mean \pm SD & $16.29 \pm 8.94$ & $13.23 \pm 9.51$ & 0.341 \\
\hline Median & $17(9-24.5)$ & $12.0(4-23.5)$ & \\
\hline
\end{tabular}

Table 3: Treatment response and outcome.

CR: Complete Response; PR: Partial Response; SD: Stable Disease; PD: Progressive Disease. 
At the end of 6 months follow-up, one more patient achieved CR while another patient disease status changed from SD to PD, so overall CR, PR, SD and PD were 13 (76.5\%),1 (5.9\%),1 (5.9\%) and $2(11.8 \%)$ in HFCRT (VMAT) arm respectively. On the other side, in CRT (VMAT) arm, two more patients converted into CR and five patients into PD so net total 11 (64.7\%) patients achieved complete response and $6(35.3 \%)$ patients disease progressed. Although, HFCRT (VMAT) arm has more proportion of patients with complete response and less patients with progressive disease in comparison to CRT (VMAT) arm, but it failed to achieve any statistical significance $(p=0.255)$.

$2(5.9 \%)$ patients out of 34 had nodal relapse out of which 1 (2.9\%) patient in hyperfractionated CCRT arm and 1 (2.9\%) patient in conventional CCRT arm who underwent salvage surgery. Two patients out of 34 patients presented with metastasis on 6 month follow up. Out of which 1 patient in hyperfractionated CCRT presented with adrenal metastasis and 1 patient in conventional CCRT arm presented with lung metastasis.

\section{Survival}

The median follow-up of surviving patients was 15 months (range from 4-30 months), two (11.8\%) patients in each arm had locoregional failure while 1 (5.9\%) patient in HFCRT (VMAT) arm developed adrenal metastasis and $2(11.8 \%)$ patients in CRT (VMAT) arm presented with lung metastasis.

In figure 2 and 3, Kaplan-Meier estimate of overall survival and disease-free survival are shown. At the last follow up, median overall survival were 17 months versus 12 months in HFCRT (VMAT) arm and in CRT (VMAT) arm respectively. On comparison, overall survival is better in HFCRT (VMAT) arm, however not statistically significant (log-rank test, $\mathrm{p}=0.341$ ). In parallel, DFS was also improved in HFCRT (VMAT) arm and continued to be better till the last follow up but difference did not reach significance (log-rank test, $\mathrm{p}=0.506$ ).

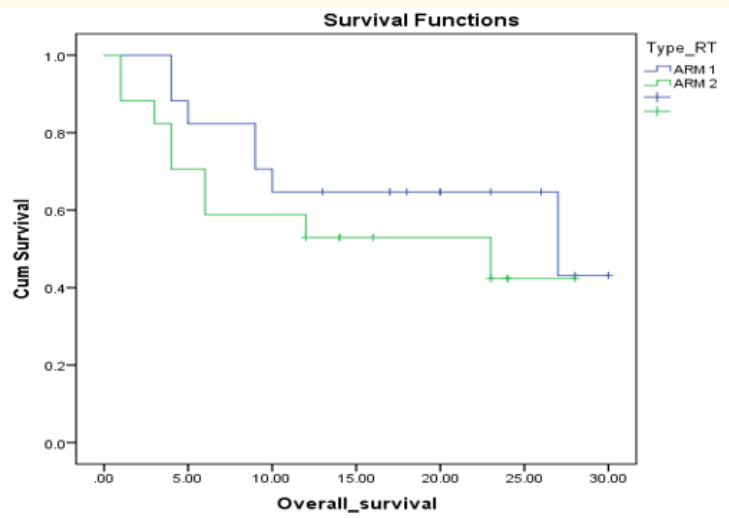

Figure 2: Kaplan-Meier curve for overall survival for both arms. Log Rank (Mantel-Cox); p = 0.341; Arm 1: HFCRT(VMAT), Arm 2: CCRT(VMAT).

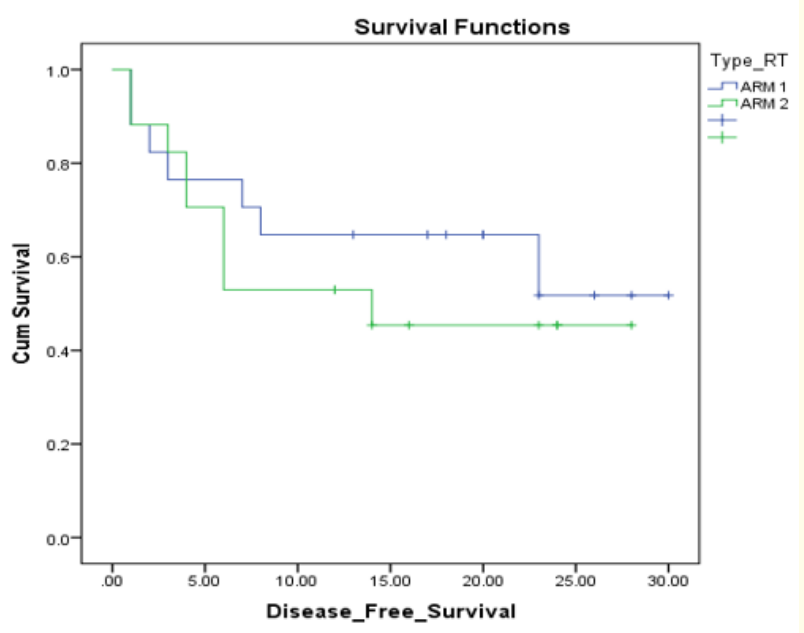

Figure 3: Kaplan-Meier curve for disease free survival for both arms. Log Rank (Mantel-Cox); $\mathrm{p}=0.506$; Arm 1: HFCRT(VMAT), Arm 2: CCRT(VMAT).

\section{Discussion}

Irrespective of the modality of primary treatment in most patients with advanced head and neck cancer, residual or local relapse represent the major treatment failure and thus, emphasizing the role of locoregional control for the patients long-term survival. With advanced stage in head and neck cancer, the causes of resistance to treatment e.g. tumor intrinsic radio-resistance, sublethal damage repair of tumor cells, hypoxia and tumor repopulation are more prevalent. So, total radiation dose, dose per fractionation and overall treatment time are important determinant in radiation treatment outcome. The current standard of care in advanced head and neck cancer is concomitant chemoradiation (CRT). Altered fractionation radiotherapy such as hyperfractionated (HF) or accelerated fractionation has been widely tried in various clinical setting to improve therapeutic ratio. HFRT enables successful dose escalation to tumor, while minimizing the effects on late-responding tissues thus, increases therapeutic ratio.

Benefit of addition of chemotherapy to HFRT in terms of improved overall survival and locoregional control have been proved in many studies including one recent metanalysis but with increased acute toxicities (like stomatitis) and late toxicities (like xerostomia) [5-7]. There have been concerns of an increase in toxicities for HNSCC patients on HFRT as observed in prior trials using 2D radiotherapy. The modern adaptive volumetric modulated arc radiotherapy (VMAT) technique offers a better conformal dose distribution and also less treatment time; thereby reduces treatment errors and toxicity to normal structures.

This study compared hyperfractionated radiotherapy with concomitant chemotherapy (HFCRT) to conventional concomitant chemoradiotherapy (CRT) in locally advanced head and neck can- 
cer via VMAT radiation technique. The hypothesis of this study was that HFCRT would have superior results to CRT and using modern technique VMAT would minimise the toxicity which is a major concern in adding chemotherapy to already toxic HFRT. As of yet no prospective randomised trial compared both radiation regimen treated by VMAT.

In the study, patients randomized into two arms of HFCRT and conventional CRT arm along with concomitant weekly cisplatin chemotherapy with VMAT technique used in both the arms. Radiation dose in HFCRT (VMAT) arm is $81.6 \mathrm{~Gy}$ in 68 fractions which is higher than 70Gy in 35 fractions of CRT (VMAT) arm as enabled by giving small dose of $1.2 \mathrm{~Gy} /$ fraction twice daily, the beauty of hyperfractionation.

All the patients in both arms were able to complete their treatment as schedule with good compliance to chemotherapy which is quite high as reported earlier on hyperfractionated radiotherapy studies done with conventional radiation techniques [5,17]. Jeremic., et al. retrospectively investigated the difference between conventionally fractionated (CF) and hyperfractionated (HF) radiation therapy (RT), with and without either daily cisplatin (CDDP) or carboplatin (CBDCA) in locally advanced HNSCC in four treatment group CF RT, CF RT plus chemotherapy (CHT), HF RT and HF RT plus CDDP and observed that HF RT was more toxic than CF RT, either alone or with concurrent CHT [17]. In the present study, correspondingly HFCRT (VMAT) arm experienced increased incidence of high grade acute skin and mucosal toxicities in comparison to CRT arm but was not statistically significant. However, there was no high grade late toxicity reported in this study, which is contrary to Jeremic., et al. result of higher incidence of xerostomia in HF RT alone and HF RT plus CDDP group. Franzese., et al. conducted study on 54 patients of advanced Oropharyngeal carcinoma treated with radical chemoradiotherapy with VMAT technique and found that there was an absence of grade 4 or more events in the acute and late treatment related toxicity with no grade 3 late skin, mucosal and taste and negligible grade 3 late dysphagia and xerostomia [18]. The explanation of no high grade late toxicity and manageable acute toxicity in HFCRT arm could be correlated with treatment of patients by VMAT radiation technique.

Katori., et al. compared hyperfractionation (HF) and conventional fractionation radiotherapy (CRT) with concurrent docetaxel, cisplatin and 5-fluorouracil (TPF) chemotherapy in 50 patients with locally advanced HNSCC. In this trial, overall clinical response rate and the pathological CR were superior in arm HFRT as compared to arm CRT with no significant difference [19]. Likewise, more patients achieved complete response rate and few patients disease progressed both at 3 months and 6 months follow-up in HFCRT (VMAT) arm in current study, but statistically insignificant.

A latest Bayesian network meta-analysis (NMA) conducted by Liu Y., et al. includes 72 trials with 21,868 patients, compared efficacy and safety of treatment of conventional fractionation (CF), conventional chemoradiotherapy (CF_CRT), hyperfractionated radiotherapy (HF), hyperfractionated chemoradiotherapy (HF_CRT), accelerated hyperfractionated radiotherapy (HART) or accelerated hyperfractionated chemoradiotherapy (HACRT). The result indicated that all treatments were associated with a significant advantage compared to CF alone concerning OS and HACRT had the best ranking for OS and locoregional control (LRC) and HF_CRT for DFS [20]. In similar fashion, Jeremic., et al. and Katori., et al. also showed hyperfractionated radiotherapy plus chemotherapy group had better OS, DFS and LRC than any other group [17,19]. In the current study, OS and DFS equivalently improved in HFCRT (VMAT) arm and DFS continued to better throughout the study. Although, OS and DFS displayed encouraging result which failed to reach significant difference.

However, the present study did not show statistical significant therapeutic benefit as per our hypothesis, could be attributed to plausible reasons, i.e., first, the hyperfractionated arm has more patients with advanced stage (IV). Secondly, the sample is small ( $\mathrm{n}$ = 34) so it is difficult to draw a significant difference between two arms of study. The other limitation is the short follow-up, with median of 15 months.

The major observations of the study were good compliance rate i.e. all patient completed their treatment as per protocol, manageable acute toxicity and no high grade late toxicity in either arm on long term follow-up. These observations favour giving hyperfractionated radiotherapy with concomitant chemotherapy by advanced radiation technique such as VMAT. However, outcome of the study could be replicated on large sample with minimal toxicity still needs to be explored.

\section{Conclusion}

In conclusion, hyperfractionated radiotherapy with concomitant chemotherapy showed an encouraging trend towards overall 
survival and disease free survival with good toxicity profile over chemoradiation. The combination of hyperfractionated radiotherapy and weekly cisplatin especially treating with modern radiation technique such as VMAT is a preferable treatment option in advanced stage head and neck cancer patients.

\section{Acknowledgements}

The authors are thankful to Vydehi Institute of Medical Sciences and Research Centre, Bangalore, India for support.

\section{Funding}

No Funding.

\section{Conflict of Interest}

The authors declare no conflicts of interest.

\section{Ethical Approval}

The study was approved by the Institutional Ethics Committee.

\section{Bibliography}

1. Report of National Cancer Registry Programme (ICMR-NCDIR), Bengaluru, India (2020).

2. Kushwaha VS., et al. "Gefitinib, Methotrexate and Methotrexate plus 5-Fluorouracil as palliative treatment in recurrent head and neck squamous cell carcinoma". Cancer Biology and Therapy 16.2 (2015): 346-351.

3. Pignon JP., et al. "Meta-analysis of chemotherapy in head and neck cancer (MACH-NC): an update on 93 randomised trials and 17,346 patients". Radiotherapy Oncology 92.1 (2009): 4-14.

4. Lacas B., et al. "Role of radiotherapy fractionation in head and neck cancers (MARCH): an updated meta-analysis". Lancet Oncology 18.9 (2017): 1221-1237.

5. Al-Saleh K., et al. "Hyperfractionated radiation therapy and concurrent chemotherapy for advanced head and neck cancer". Gulf Journal of Oncology 9 (2011): 12-19.

6. Jeremic B., et al. "Hyperfractionated radiation therapy with or without concurrent low-dose daily cisplatin in locally advanced squamous cell carcinoma of the head and neck: a prospective randomized trial". Journal of Clinical Oncology 18.7 (2000): 1458-1464.
7. Brizel DM., et al. "Hyperfractionated irradiation with or without concurrent chemotherapy for locally advanced head and neck cancer". The New England Journal of Medicine 338.25 (1998): 1798-1804.

8. Haussmann J., et al. "Addition of chemotherapy to hyperfractionated radiotherapy in advanced head and neck cancer-a meta-analysis". Strahlentherapie und Onkologie 195.12 (2019): 1041-1049.

9. Fu KK., et al. "A Radiation Therapy Oncology Group (RTOG) phase III randomized study to compare hyperfractionation and two variants of accelerated fractionation to standard fractionation radiotherapy for head and neck squamous cell carcinomas: first report of RTOG 9003". International Journal of Radiation Oncology*Biology*Physics 48.1 (2000): 7-16.

10. Maguire PD., et al. "Phase II trial of hyperfractionated intensity-modulated radiation therapy and concurrent weekly cisplatin for Stage III and IVa head-and-neck cancer". International Journal of Radiation Oncology*Biology*Physics 79.4 (2011): 1081-1088.

11. van der Veen J and Nuyts S. "Can Intensity-Modulated-Radiotherapy Reduce Toxicity in Head and Neck Squamous Cell Carcinoma?" Cancers (Basel) 9.10 (2017): 135.

12. Franzese C., et al. "Toxicity profile and early clinical outcome for advanced head and neck cancer patients treated with simultaneous integrated boost and volumetric modulated arc therapy". Radiation Oncology 10 (2015): 224.

13. Moncharmont C., et al. "Real-life efficacy of volumetric modulated arc therapy in head and neck squamous cell carcinoma". European Annals of Otorhinolaryngology, Head and Neck Diseases 134.3 (2017): 165-169.

14. Amin MB., et al. "The Eighth Edition AJCC Cancer Staging Manual: Continuing to build a bridge from a population-based to a more "personalized" approach to cancer staging". CA: A Cancer Journal for Clinicians 67.2 (2017): 93-99.

15. Schwartz LH., et al. "RECIST 1.1-Update and clarification: From the RECIST committee". European Journal of Cancer 62 (2016): 132-137.

16. Cox JD., et al. "Toxicity criteria of the Radiation Therapy Oncology Group (RTOG) and the European Organization for Research and Treatment of Cancer (EORTC)". International Journal of Radiation Oncology*Biology*Physics 31.5 (1995): 1341-1346. 
17. Jeremic B., et al. "Radiation therapy with or without concurrent low-dose daily chemotherapy in locally advanced, nonmetastatic squamous cell carcinoma of the head and neck". Journal of Clinical Oncology 22.17 (2004): 3540-3548.

18. Franzese C., et al. "Treatment: Outcome and Toxicity of Volumetric Modulated Arc Therapy in Oropharyngeal Carcinoma". Anticancer Research 36.7 (2016): 3451-3457.

19. Katori H., et al. "Comparison of hyperfractionation and conventional fractionation radiotherapy with concurrent docetaxel, cisplatin and 5-fluorouracil (TPF) chemotherapy in patients with locally advanced squamous cell carcinoma of the head and neck (SCCHN)". Cancer Chemotherapy and Pharmacology 60.3 (2007): 399-406.

20. Liu Y., et al. "Altered fractionation radiotherapy with or without chemotherapy in the treatment of head and neck cancer: a network meta-analysis". OncoTargets and Therapy 11 (2018): 5465-5483.

\section{Assets from publication with us}

- Prompt Acknowledgement after receiving the article

- Thorough Double blinded peer review

- Rapid Publication

- Issue of Publication Certificate

- High visibility of your Published work

Website: www.actascientific.com/

Submit Article: www.actascientific.com/submission.php

Email us: editor@actascientific.com

Contact us: +919182824667

Citation: Vandana S Kushwaha. "Comparison of Hyperfractionated Radiotherapy with Concomitant Chemotherapy and Conventional Chemoradiotherapy in Locally Advanced Head and Neck Cancer Treated with Volumetric Modulated Arc Technique (VMAT)". Acta Scientific Cancer Biology 5.5 (2021): $02-11$. 